\title{
Manganese and Extracellular Superoxide Dismutase Polymorphisms and Risk for Asbestosis
}

\author{
Alenka Franko, ${ }^{1}$ Metoda Dodič-Fikfak, ${ }^{1}$ Niko Arnerić, ${ }^{1}$ and Vita Dolžan ${ }^{2}$ \\ ${ }^{1}$ Clinical Institute of Occupational Medicine, University Medical Centre, Ljubljana, Poljanski nasip 58, 1000 Ljubljana, Slovenia \\ ${ }^{2}$ Institute of Biochemistry, Faculty of Medicine, University of Ljubljana, Vrazov trg 2, 1000 Ljubljana, Slovenia \\ Correspondence should be addressed to Vita Dolžan, vita.dolzan@mf.uni-lj.si
}

Received 9 February 2009; Revised 8 May 2009; Accepted 24 May 2009

Recommended by M. Ilyas Kamboh

Manganese and extracellular superoxide dismutases (SOD2 and SOD3) are part of the enzymatic defence against reactive oxygen species, which are involved in the pathogenesis of asbestosis. This study investigates whether SOD2Ala -9Val and SOD3 Arg213Gly genetic polymorphisms represent risk factors for asbestosis in workers exposed to asbestos. The study included 262 cases with asbestosis and 265 controls with no asbestos-related disease. Cumulative asbestos exposure was calculated for each subject. A real-time PCR assay was introduced for genotyping. Logistic regression analysis was used to assess asbestosis risk. Asbestosis was associated with the homozygous SOD2 -9Ala/Ala genotype (OR $=1.50,95 \% \mathrm{CI} 1.01-2.24)$, whereas the association for the SOD3 $\mathrm{Arg} / \mathrm{Gly}$ genotype was not significant $(\mathrm{OR}=1.63,95 \% \mathrm{CI} 0.62-4.27)$. The finding that the SOD2 -9Ala/Ala genotype increases the risk for asbestosis indicates that, in addition to asbestos exposure, genetic factors may also have a significant influence on the development of asbestosis.

Copyright (c) 2009 Alenka Franko et al. This is an open access article distributed under the Creative Commons Attribution License, which permits unrestricted use, distribution, and reproduction in any medium, provided the original work is properly cited.

\section{Introduction}

Exposure to asbestos fibres has been shown to cause asbestosis, pleural diseases such as pleural plaques, lung cancer, malignant mesothelioma and some other types of cancers [1-3]. One of the most frequent diseases caused by inhalation of asbestos is asbestosis, an interstitial pulmonary process that slowly develops into diffuse pulmonary fibrosis [4]. Although the causal relationship between asbestos exposure and asbestosis has been well proved, little is still known about the genetic factors that may influence the development of this disease $[2,5]$. At present it is suggested that both environmental and genetic factors may play an important role in the development of asbestosis $[2,6,7]$. The gene-environmental interaction studies published so far have mostly focused on the genes coding for xenobiotic metabolizing enzymes [2, 5-9].

Numerous studies have suggested the central role of reactive oxygen and nitrogen species (ROS and RNS) in fibre-induced toxicity and development of asbestosis [1, $10,11]$. The most important reactive metabolites in the pathogenesis of asbestos-related lung diseases are superoxide anion $\left(\mathrm{O}_{2}{ }^{--}\right)$, hydrogen peroxide $\left(\mathrm{H}_{2} \mathrm{O}_{2}\right)$, hydroxyl radical $\left(\mathrm{OH}^{\bullet}\right)$ and nitric oxide (NO) $[10,12,13]$.

Human tissues contain specific enzyme systems to detoxify ROS and RNS. Superoxide dismutases (SODs) together with catalase and glutathione peroxidases constitute a primary defence against oxidative stress $[10,14,15]$. SODs catalyze the conversion of superoxide anion $\left(\mathrm{O}_{2}{ }^{--}\right)$ to hydrogen peroxide $\left(\mathrm{H}_{2} \mathrm{O}_{2}\right)$ and oxygen $\left(\mathrm{O}_{2}\right)$ [14, 16]. Manganese SOD (MnSOD or SOD2) is a manganese (Mn)containing enzyme localized in mitochondria, whereas extracellular SOD (ECSOD or SOD3), which contains copper $(\mathrm{Cu})$ and zinc $(\mathrm{Zn})$, is predominantly found in extracellular space $[14,15]$.

In humans, SOD2 is encoded by the SOD2 gene localized to chromosome 6 (region 6q25), and SOD3 by the SOD3 gene, which has been localized to chromosome 4 (region $4 \mathrm{p}$ q21) $[15,17,18]$. The genes coding for SOD2 and SOD3 are polymorphic. The most common functional polymorphism of the SOD2 gene is cytosine (C) to thymine (T) substitution (201C>T, rs4880), which results in alanine (Ala) to valine (Val) amino acid change at position -9 of the mitochondrial targeting sequence (SOD2 Ala-9Val) [19, 20]. It has 
been suggested that this polymorphism alters the secondary structure of the protein, and hence may affect the efficiency of transport of the SOD2 into the mitochondria, where it would be biologically available $[19,20]$. Sutton et al. demonstrated that Ala-containing SOD2 is actively targeted into the mitochondrial matrix, whereas the Val-containing variant is partially arrested within the inner mitochondrial membrane [21]. The Ala-variant also resulted in 4-fold higher levels of the mature exogenous protein and SOD2 activity than the Val-variant [21]. The frequency of each allele is around $50 \%$ in Caucasian populations [22], whereas the Val allele is more frequent in Japan (87.9\%) [19]. Among asbestos-related diseases, the SOD2 $-9 \mathrm{Val} / \mathrm{Val}$ genotype was associated with increased lung cancer risk in individuals with a low asbestos exposure score [23], whereas another study found an increased risk of mesothelioma in subjects with the SOD2 -9Ala/Ala genotype [24]. In contrast, a study by Hirvonen et al. suggested no major modifying role for SOD2 polymorphism in asbestos-related pulmonary disorders [9].

SOD3 binds lung matrix components and inhibits their fragmentation in response to oxidative stress $[25,26]$. In the SOD3 gene, a cytosine (C) to guanine $(\mathrm{G})$ substitution (896C > G, rs1799895) causing an amino acid change from arginine (Arg) to glycine (Gly) at position 213 has been reported (Arg213Gly) [15, 27, 28]. Although this polymorphism is rare, occurring in 2 to $6 \%$ of various populations $[15,27,28]$, it causes an 8 - to 15 -fold increase in the concentration of plasma SOD3 levels due to impaired binding to the extracellular matrix $[27,28]$. To our knowledge, the influence of SOD3 Arg213Gly polymorphism on the risk of developing asbestosis in workers exposed to asbestos has not been studied so far. Most of the data come from studies on SOD3 transgenic $[29,30]$ and knockout mice $[31,32]$ that clearly demonstrate the importance of SOD3 in lung protection. Enhanced lung damage, inflammation, and fibrosis were observed in SOD3 knockout mice compared to wild-type mice in asbestos-induced lung injuries $[25,32,33]$.

To study the influence of genetic polymorphisms of some of the most important metabolic enzymes on the risk for asbestosis and possible interaction with asbestos exposure, the authors designed a series of molecular epidemiological studies $[6,7]$. The basic aim of this study was to investigate the influence of the SOD2 Ala -9Val and SOD3 Arg213Gly genetic polymorphisms on the risk for developing asbestosis in workers occupationally exposed to asbestos.

\section{Methods}

In a nested case-control study, all 356 subjects diagnosed with asbestosis were first selected from the cohort of 2080 workers that were presented at the State Board for the Recognition of Occupational Asbestos Diseases from 1 January 1998 to 31 December 2003. For each case, one control with no asbestos-related disease was recruited from the same cohort using gender and date of birth as matching factors.
From the selected subjects, 2 cases and 9 controls developed cancer in the period between the recognition of the occupational disease and the beginning of the study, 40 cases and 29 controls died during the same period, and 52 cases and 53 controls refused to participate, so the final number of cases was $262(73.6 \%)$ and that of controls 265 (74.4\%).

Information about smoking, including duration of smoking and the number of pack-years of smoking, was obtained for all subjects during an interview using a standardized questionnaire $[3,34]$.

All subjects that participated in the study worked at the Salonit Anhovo asbestos cement manufacturing plant in Anhovo, Slovenia and were occupationally exposed to asbestos [3]. For each case and control included in this study, the data on cumulative asbestos exposure were available from the previous study [3]. The methodology of cumulative asbestos exposure calculation has already been described in detail in previous papers $[3,6]$.

The diagnosis of asbestosis or "no asbestos-related disease" was confirmed by two groups of experts from the State Board for the Recognition of Occupational Asbestos Diseases that operate at the Clinical Institute of Occupational Medicine; each group consisted of an occupational physician, a pulmonologist, and a radiologist. The diagnostic criteria were based on the Helsinki Criteria for Diagnosis and Attribution of Asbestos Diseases [35] and on the American Thoracic Society recommendations [36].

For genetic analysis, capillary blood samples from the fingertips of all subjects were collected on FTA Mini Cards (Whatman Bioscience) and genomic DNA was isolated as previously described [6].

To determine SOD2 Ala -9Val and SOD3 Arg213Gly polymorphisms, TaqMan SNP genotyping assays were used (C_8709053_10 and C_2307506_10, resp., AB assay, Applied Biosystems, Foster City, Calif. USA). Real-time PCR was performed under universal conditions on ABI 7500 (Applied Biosystems, Foster City, Calif. USA). TaqMan SNP genotyping assays were validated on 100 samples genotyped by the PCR-RFLP method. To determine SOD2 Ala -9Val polymorphism, the region encompassing the polymorphic site was amplified and PCR products were digested withNgoM IV (New England Biolabs), as previously described [37]. To determine SOD3 Arg213Gly polymorphism, the corresponding PCR products were digested with $\mathrm{MwoI}$ (New England Biolabs), as previously described [38].

Among statistical methods, standard descriptive statistics were first performed. Next, $t$-tests for differences of means of variables between the cases and controls were calculated, and $\chi^{2}$ tests were used to determine whether the observed differences in proportions between the study groups were statistically significant. To assess the causal relationship between asbestosis, genotypes, cumulative asbestos exposure, and standard confounders (age, sex), univariate logistic regression was first used, followed by multivariate logistic regression modelling. A possible synergistic effect between genotypes and cumulative asbestos exposure was investigated by using dummy variables. $P$-values less than 0.05 were considered statistically significant. 
TABLE 1: Smoking and cumulative asbestos exposure in cases and controls.

\begin{tabular}{lccccccccc}
\hline & \multicolumn{3}{c}{ Cases $(n=262)$} & \multicolumn{3}{c}{ Controls $(n=265)$} & Test & $P$-value \\
\hline Smoking (ever/never smokers, $n)$ & \multicolumn{2}{c}{$117 / 145$} & & $120 / 145$ & $\chi^{2}=0.01$ & .919 \\
\hline & Mean & SD & Range & Mean & SD & Range & \\
\hline Years of smoking & 25.92 & 13.37 & $0.01-60.00$ & 22.90 & 12.90 & $1.00-61.00$ & $t=1.77$ & .078 \\
Pack-years of smoking & 21.92 & 15.95 & $0.15-66.25$ & 20.99 & 16.37 & $0.05-90.00$ & $t=0.44$ & .659 \\
\hline Cumulative asbestos exposure (fibres $/ \mathrm{cm}^{3}$-years) & 37.67 & 86.43 & $0.01-869.72$ & 11.23 & 23.47 & $0.01-149.21$ & $t=4.78$ & .000 \\
\hline
\end{tabular}

TABLE 2: Frequencies of SOD2 and SOD3 genotypes in cases and controls.

\begin{tabular}{|c|c|c|c|c|c|c|}
\hline \multirow[b]{2}{*}{ SOD2 genotype } & \multicolumn{2}{|c|}{ Cases $(n=258)$} & \multicolumn{2}{|c|}{ Controls $(n=261)$} & \multirow[b]{2}{*}{$\chi^{2}$} & \multirow[b]{2}{*}{$P$-value } \\
\hline & $n$ & $\%$ & $n$ & $\%$ & & \\
\hline Ala/Ala & 75 & 29.0 & 56 & 21.4 & & \\
\hline $\mathrm{Ala} / \mathrm{Val}$ & 116 & 45.0 & 139 & 53.3 & $4.79^{*}$ & .029 \\
\hline $\mathrm{Val} / \mathrm{Val}$ & 67 & 26.0 & 66 & 25.3 & $1.25^{\dagger}$ & .263 \\
\hline \multirow[t]{2}{*}{$\mathrm{Ala} / \mathrm{Val}+\mathrm{Val} / \mathrm{Val}$} & 183 & 71.0 & 205 & 78.6 & $3.99^{\ddagger}$ & .046 \\
\hline & \multicolumn{2}{|c|}{ Cases $(n=258)$} & \multicolumn{2}{|c|}{ Controls $(n=263)$} & & \\
\hline SOD3 genotype & $n$ & $\%$ & $n$ & $\%$ & $\chi^{2}$ & $P$-value \\
\hline Arg/Arg & 247 & 95.7 & 256 & 97.3 & & \\
\hline Arg/Gly & 11 & 4.3 & 7 & 2.7 & $1.00^{\S}$ & .317 \\
\hline Gly/Gly & 0 & 0 & 0 & 0 & & \\
\hline
\end{tabular}

${ }^{*} \chi^{2}$ calculated for Ala/Ala versus Ala/Val

$+\chi^{2}$ calculated for Ala/Ala versus Val/Val

${ }^{\ddagger} \chi^{2}$ calculated for Ala/Ala versus Ala/Val $+\mathrm{Val} / \mathrm{Val}$

$\S \chi^{2}$ calculated for Arg/Arg versus Arg/Gly.

The study was approved by the Slovenian Ethics Committee for Research in Medicine and was carried out in line with the Helsinki Declaration.

\section{Results}

There was no statistical difference in gender between the cases and controls. Among the 262 cases there were 186 men and 76 women, and among the 265 controls 183 men and 82 women $\left(\chi^{2}=0.24, P=.628\right)$. On average, cases were 61 and controls 57 years old, showing a statistical difference between them $(t=5.18, P=.000)$. No difference in smoking was observed between the cases and controls (Table 1).

As expected, the mean cumulative asbestos exposure was higher among the cases than the controls $(t=4.78, P=.000)$ (Table 1).

All of the subjects were genotyped for SOD2 Ala $-9 \mathrm{Val}$ and SOD 3 Arg213Gly polymorphisms, although the amplification of the SOD2 gene failed in 4 cases and 4 controls, and that of the SOD3 gene in 4 cases and 2 controls.

Three different SOD2 genotypes (Ala/Ala, Ala/Val, and $\mathrm{Val} / \mathrm{Val}$ ) and two SOD3 genotypes (Arg/Arg and Arg/Gly) were detected. The SOD2 -9Ala/Ala genotype was observed in $29.0 \%$ of cases and $21.4 \%$ of controls, Ala/ $\mathrm{Val}$ in $45.0 \%$ of cases and $53.3 \%$ of controls, and $\mathrm{Val} / \mathrm{Val}$ in $26.0 \%$ of cases and $25.3 \%$ of controls. The SOD3 Arg/Arg genotype was found in $95.7 \%$ of cases and $97.3 \%$ of controls, and the $\mathrm{Arg} / \mathrm{Gly}$ genotype was observed in $4.3 \%$ of cases and $2.7 \%$ of controls. Combining polymorphic SOD2 genotypes, the frequency of the -9Ala/Ala genotype versus the frequency of the Ala/Val and Val/Val genotypes was significantly higher in the cases than in the controls $\left(\chi^{2}=3.99, P=.046\right)$ (Table 2). In contrast, no significant difference was found in the frequency of the SOD3 Arg/Arg genotype compared to the frequency of the Arg/Gly genotype $\left(\chi^{2}=1.00, P=.317\right)$ (Table 2).

In subsequent logistic regression analysis, no association was observed between asbestosis and ever/never smoking $(\mathrm{OR}=0.98,95 \%$ CI 0.69-1.39). Knowing that cumulative asbestos exposure was skewed to the right, logarithmically transformed cumulative asbestos exposure was calculated, giving an OR of asbestosis of 3.21 (95\% CI 2.43-4.23).

Analyzing the association between asbestosis and SOD2 genotypes, the OR of asbestosis was 1.61 (95\% CI 1.05-2.46) for the -9Ala/Ala genotype versus the Ala/Val genotype, 1.32 (95\% CI 0.81-2.14) for the Ala/Ala genotype versus the $\mathrm{Val} / \mathrm{Val}$ genotype, and 1.50 (95\% CI 1.01-2.24) for the Ala/Ala genotype versus combined $\mathrm{Ala} / \mathrm{Val}$ and $\mathrm{Val} / \mathrm{Val}$ genotypes. The OR of asbestosis calculated for the SOD3 Arg/Gly genotype compared to the Arg/Arg genotype was 1.63 (95\% CI 0.62-4.27).

Using multivariate logistic regression modelling, the risk of asbestosis for the SOD2 -9Ala/Ala genotype versus combined $\mathrm{Ala} / \mathrm{Val}$ and $\mathrm{Val} / \mathrm{Val}$ genotypes did not change considerably after adjustment by gender, age, smoking, and cumulative asbestos exposure (Table 3 ). The results showed that gender, age, and smoking did not substantially influence 
TABLE 3: Risk of asbestosis for the SOD2 -9Ala/Ala versus combined Ala/Val and Val/Val genotypes and SOD3 Arg/Gly genotype compared to Arg/Arg genotype after adjustment by gender, age, smoking, and cumulative asbestos exposure.

\begin{tabular}{lcccr}
\hline & \multicolumn{2}{c}{ SOD2 -9Ala/Ala } & \multicolumn{2}{c}{ SOD3 Arg/Gly } \\
& OR & $95 \%$ CI & OR & 1.63 \\
Unadjusted & 1.50 & $1.01-2.24$ & & \\
Adjusted by & & & 1.61 & $0.62-4.27$ \\
$\quad$ Gender & 1.49 & $1.00-2.23$ & 1.49 & $0.61-4.22$ \\
Age & 1.46 & $0.97-2.19$ & 1.65 & $0.56-3.96$ \\
Smoking (ever/never) & 1.49 & $1.00-2.23$ & 2.07 & $0.63-4.32$ \\
Cumulative exposure & 1.48 & $0.96-2.28$ & $0.72-5.94$ \\
\hline
\end{tabular}

the risk of asbestosis for the SOD3 Arg/Gly compared to Arg/Arg genotype either, but the involvement of cumulative asbestos exposure changed the OR from 1.63 (95\% CI $0.62-$ 4.27 ) to 2.07 ( $95 \%$ CI $0.72-5.94)$, indicating a possible confounding effect of cumulative asbestos exposure (Table 3 ). To test the possible interaction between asbestos exposure and the SOD3 genotype, a dummy variable was created by multiplying the SOD3 Arg/Gly genotype and cumulative asbestos exposure. No synergistic effect was found $(\mathrm{OR}=$ $0.61, P=.456)$. When the SOD2 and SOD3 genotypes were simultaneously introduced into logistic regression analysis, the OR of asbestosis was 1.51 (95\% CI 1.01-2.25) for the SOD2 -9Ala/Ala genotype and 1.61 (95\% CI 0.61-4.24) for the SOD3 Arg/Gly genotype, showing independent activity of the two.

\section{Discussion}

Increasing evidence suggests that both asbestos exposure and genetic factors play an important role in the development of asbestosis [2, 6-8], which is known to be among the most frequent asbestos-related diseases. The key finding of this study is that the SOD2 -9Ala/Ala genotype increases risk of asbestosis. Although the function of SOD2 Ala -9 Val polymorphism requires further investigation and elucidation, it has been suggested that human SOD2 with Ala in the mitochondrial targeting sequence allows more efficient import into the mitochondrial matrix and is therefore presumed to result in higher levels of the mature SOD2 and enzyme activity than the Val-variant $[19,21]$. In line with these observations, it could be predicted that having the SOD2 -9Ala/Ala genotype is beneficial [39]. However, several epidemiological studies have indicated an increased risk of developing diseases in individuals carrying this genotype $[22,24,40,41]$. This discovery is in agreement with the results of our study. In addition, it has been suggested that SOD2 may play a dual role in relation to ROS [22]. On the one hand, SOD2 is considered to be a detoxifying enzyme that removes superoxide anion $[14,16]$. On the other hand, it has been suggested that $\mathrm{H}_{2} \mathrm{O}_{2}$ generated by SOD2 is toxic because it can be converted to the more toxic $\mathrm{OH}^{\bullet}$ radical if not removed quickly and efficiently enough by glutathione peroxidase and catalase [21, 42]. An elevated level of SOD2 activity could therefore result in increased production of $\mathrm{H}_{2} \mathrm{O}_{2}$ and $\mathrm{OH}^{\bullet}$ radicals, which can cause cell injury and consequently a higher risk of developing diseases [42]. In line with these reports and the assumption that individuals with the SOD2 -9Ala/Ala genotype may have higher SOD2 enzyme activity and therefore likely elevated levels of $\mathrm{H}_{2} \mathrm{O}_{2}$ and $\mathrm{OH}^{*}$ radicals, which are known to be involved in the pathogenesis of asbestosis, our finding of an increased risk of asbestosis in subjects with the SOD2 -9Ala/Ala genotype may be considered biologically plausible.

In contrast to the observations of the present study, Hirvonen et al. suggested no major modifying role for SOD2 polymorphism in asbestos-related pulmonary disorders in a study based on 20 subjects with mesothelioma and 41 subjects with asbestosis or/and pleural plaques [9]. However, Landi et al. found an increased risk of mesothelioma in subjects with the SOD2 -9Ala/Ala genotype, which very likely agrees with the findings of this study [24].

A slightly elevated risk of asbestosis was also observed for the SOD3 Arg/Gly genotype, but the result was not significant. Despite the higher frequency of the SOD3 Arg/Gly genotype in subjects with asbestosis compared to the controls, no significant difference was observed due to the lack of power. However, the results of several studies demonstrated that SOD3 knockout mice show enhanced lung damage, inflammation, and fibrosis compared to wildtype mice in asbestos-induced lung injuries $[25,32,33,43]$. In line with these reports, SOD3 polymorphism might play a role in developing asbestosis and should therefore be further investigated in association with this disease. The risk of asbestosis for the SOD3 Arg/Gly genotype increased slightly after involvement of cumulative asbestos exposure. No synergistic effect was found, but cumulative asbestos exposure acted as a confounder.

The findings of the current study were not biased by genetic heterogeneity because all of the subjects were recruited in a small geographic area with an ethnically homogenous population. There were also no differences in the cumulative exposure or diagnosis of cancer between the participants and subjects that did not want to take part in the study, died or developed a malignant disease in the period between the recognition of occupational disease and beginning of this study.

Overall, this study found evidence of an association between asbestosis and SOD2 Ala -9Val genetic polymorphism. The results of our previous studies showed 
a decreased risk of asbestosis in individuals with the glutathione S-transferase T1-null genotype [6] and an increased risk in subjects carrying the glutathione S-transferase P1 genotype coding for an enzyme with high conjugation capacity [7]. All of these results suggest that genetic factors may have a significant influence on the development of asbestosis. These findings should also be seriously considered in future research in association with other asbestos-related diseases. Furthermore, to our knowledge this is the first study investigating the role of SOD3 genetic variants in relation to asbestosis in workers occupationally exposed to asbestos, which is a topic that demands further investigation and elucidation.

\section{Acknowledgments}

The authors declare no conflict of interest. This research was financially supported by the Ministry of Education, Science, and Sport of Slovenia (Grants no. L3-9129 and P1-0170).

\section{References}

[1] B. T. Mossman, J. Bignon, M. Corn, A. Seaton, and J. B. L. Gee, "Asbestos: scientific developments and implications for public policy," Science, vol. 247, no. 4940, pp. 294-301, 1990.

[2] C. M. Smith, K. T. Kelsey, J. K. Wiencke, K. Leyden, S. Levin, and D. C. Christiani, "Inherited glutathione-S-transferase deficiency is a risk factor for pulmonary asbestosis," Cancer Epidemiology Biomarkers and Prevention, vol. 3, no. 6, pp. 471477, 1994.

[3] M. Dodič Fikfak, D. Kriebel, M. M. Quinn, E. A. Eisen, and D. H. Wegman, "A case control study of lung cancer and exposure to chrysotile and amphibole at a Slovenian asbestos-cement plant," Annals of Occupational Hygiene, vol. 51, no. 3, pp. 261268, 2007.

[4] G. A. Peters and B. J. Peters, "Medical aspects," in Sourcebook on Asbestos Diseases: Medical, Legal, and Engineering Aspects, G. A. Peters and B. J. Peters, Eds., pp. B2-B6, Gerland STPM Press, New York, NY, USA, 1980.

[5] K. Jakobsson, A. Rannung, A.-K. Alexandrie, L. Rylander, M. Albin, and L. Hagmar, "Genetic polymorphism for glutathione-S-transferase $\mu$ in asbestos cement workers," Occupational and Environmental Medicine, vol. 51, no. 12, pp. 812-816, 1994.

[6] A. Franko, M. Dodič Fikfak, N. Arneric, and V. Dolzan, "Glutathione S-transferases GSTM1 and GSTT1 polymorphisms and asbestosis," Journal of Occupational and Environmental Medicine, vol. 49, no. 6, pp. 667-671, 2007.

[7] A. Franko, V. Dolzan, N. Arneric, and M. Dodič Fikfak, "The influence of genetic polymorphisms of GSTP1 on the development of asbestosis," Journal of Occupational and Environmental Medicine, vol. 50, no. 1, pp. 7-12, 2008.

[8] K. T. Kelsey, H. H. Nelson, J. K. Wiencke, C. M. Smith, and S. Levin, "The glutathione S-transferase $\theta$ and $\mu$ deletion polymorphisms in asbestosis," American Journal of Industrial Medicine, vol. 31, no. 3, pp. 274-279, 1997.

[9] A. Hirvonen, J. Tuimala, T. Ollikainen, K. Linnainmaa, and V. Kinnula, "Manganese superoxide dismutase genotypes and asbestos-associated pulmonary disorders," Cancer Letters, vol. 178, no. 1, pp. 71-74, 2002.
[10] V. L. Kinnula, "Oxidant and antioxidant mechanisms of lung disease caused by asbestos fibres," European Respiratory Journal, vol. 14, no. 3, pp. 706-716, 1999.

[11] V. Castranova, "Role of nitric oxide in the progression of pneumoconiosis," Biochemistry, vol. 69, no. 1, pp. 32-37, 2004.

[12] S. A. Weitzman and P. Graceffa, "Asbestos catalyzes hydroxyl and superoxide radical generation from hydrogen peroxide," Archives of Biochemistry and Biophysics, vol. 228, no. 1, pp. 373-376, 1984.

[13] D. W. Kamp, P. Graceffa, W. A. Pryor, and S. A. Weitzman, "The role of free radicals in asbestos-induced diseases," Free Radical Biology and Medicine, vol. 12, no. 4, pp. 293-315, 1992.

[14] B. Halliwell and J. M. C. Gutteridge, "Antioxidant defences," in Free Radicals in Biology and Medicine, B. Halliwell and J. M. C. Gutteridge, Eds., pp. 105-146, Oxford University Press, Oxford, UK, 3rd edition, 1999.

[15] I. N. Zelko, T. J. Mariani, and R. J. Folz, "Superoxide dismutase multigene family: a comparison of the CuZn-SOD (SOD1), $\mathrm{Mn}-\mathrm{SOD}$ (SOD2), and EC-SOD (SOD3) gene structures, evolution, and expression," Free Radical Biology and Medicine, vol. 33, no. 3, pp. 337-349, 2002.

[16] J. M. McCord and I. Fridovich, "Superoxide dismutase. An enzymic function for erythrocuprein (hemocuprein)," The Journal of Biological Chemistry, vol. 244, no. 22, pp. 60496055, 1969.

[17] S. L. Church, J. W. Grant, E. U. Meese, and J. M. Trent, "Sublocalization of the gene encoding manganese superoxide dismutase (MnSOD/SOD29 to 6 q25 by fluorescence in situ hybridization and somatic cell hybrid mapping," Genomics, vol. 14, no. 3, pp. 823-825, 1992.

[18] D. J. Hendrickson, J. H. Fisher, C. Jones, and Y.-S. Ho, "Regional localization of human extracellular superoxide dismutase gene to 4pter-q21," Genomics, vol. 8, no. 4, pp. 736$738,1990$.

[19] S. Shimoda-Matsubayashi, H. Matsumine, T. Kobayashi, Y. Nakagawa-Hattori, Y. Shimizu, and Y. Mizuno, "Structural dimorphism in the mitochondrial targeting sequence in the human manganese superoxide dismutase gene. A predictive evidence for conformational change to influence mitochondrial transport and a study of allelic association in Parkinson's disease," Biochemical and Biophysical Research Communications, vol. 226, no. 2, pp. 561-565, 1996.

[20] J. S. Rosenblum, N. B. Gilula, and R. A. Lerner, "On signal sequence polymorphisms and diseases of distribution," Proceedings of the National Academy of Sciences of the United States of America, vol. 93, no. 9, pp. 4471-4473, 1996.

[21] A. Sutton, A. Imbert, A. Igoudjil, et al., "The manganese superoxide dismutase Ala16Val dimorphism modulates both mitochondrial import and mRNA stability," Pharmacogenetics and Genomics, vol. 15, no. 5, pp. 311-319, 2005.

[22] C. B. Ambrosone, J. L. Freudenheim, P. A. Thompson, et al., "Manganese superoxide dismutase (MnSOD) genetic polymorphisms, dietary antioxidants, and risk of breast cancer," Cancer Research, vol. 59, no. 3, pp. 602-606, 1999.

[23] L. I. Wang, D. Neuberg, and D. C. Christiani, "Asbestos exposure, manganese superoxide dismutase (MnSOD) genotype, and lung cancer risk," Journal of Occupational and Environmental Medicine, vol. 46, no. 6, pp. 556-564, 2004.

[24] S. Landi, F. Gemignani, M. Neri, et al., "Polymorphisms of glutathione-S-transferase M1 and manganese superoxide dismutase are associated with the risk of malignant pleural mesothelioma," International Journal of Cancer, vol. 120, no. 12, pp. 2739-2743, 2007. 
[25] F. Gao, V. L. Kinnula, M. Myllärniemi, and T. D. Oury, "Extracellular superoxide dismutase in pulmonary fibrosis," Antioxidants and Redox Signaling, vol. 10, no. 2, pp. 343-354, 2008.

[26] C. R. Kliment, J. M. Tobolewski, M. L. Manni, R. J. Tan, J. Enghild, and T. D. Oury, "Extracellular superoxide dismutase protects against matrix degradation of heparan sulfate in the lung," Antioxidants and Redox Signaling, vol. 10, no. 2, pp. 261-268, 2008.

[27] R. J. Folz, L. Peno-Green, and J. D. Crapo, "Identification of a homozygous missense mutation (Arg to Gly) in the critical binding region of the human EC-SOD gene (SOD3) and its association with dramatically increased serum enzyme levels," Human Molecular Genetics, vol. 3, no. 12, pp. 2251-2254, 1994.

[28] J. Sandström, P. Nilsson, K. Karlsson, and S. L. Marklund, "10-fold increase in human plasma extracellular superoxide dismutase content caused by a mutation in heparin-binding domain," The Journal of Biological Chemistry, vol. 269, no. 29, pp. 19163-19166, 1994.

[29] R. J. Folz, A. M. Abushamaa, and H. B. Suliman, "Extracellular superoxide dismutase in the airways of transgenic mice reduces inflammation and attenuates lung toxicity following hyperoxia," The Journal of Clinical Investigation, vol. 103, no. 7, pp. 1055-1066, 1999.

[30] R. P. Bowler, M. Nicks, K. Warnick, and J. D. Crapo, "Role of extracellular superoxide dismutase in bleomycin-induced pulmonary fibrosis," American Journal of Physiology, vol. 282, no. 4, pp. L719-L726, 2002.

[31] L. M. Carlsson, J. Jonsson, T. Edlund, and S. L. Marklund, "Mice lacking extracellular superoxide dismutase are more sensitive to hyperoxia," Proceedings of the National Academy of Sciences of the United States of America, vol. 92, no. 14, pp. 6264-6268, 1995.

[32] C. L. Fattman, L.-Y. Chang, T. A. Termin, L. Petersen, J. J. Enghild, and T. D. Oury, "Enhanced bleomycin-induced pulmonary damage in mice lacking extracellular superoxide dismutase," Free Radical Biology and Medicine, vol. 35, no. 7, pp. 763-771, 2003.

[33] C. L. Fattman, R. J. Tan, J. M. Tobolewski, and T. D. Oury, "Increased sensitivity to asbestos-induced lung injury in mice lacking extracellular superoxide dismutase," Free Radical Biology and Medicine, vol. 40, no. 4, pp. 601-607, 2006.

[34] B. G. Ferris, "Epidemiology Standardization Project (American Thoracic Society)," American Review of Respiratory Disease, vol. 118, no. 6, part 2, pp. 1-120, 1978.

[35] D. W. Henderson, J. Rantanen, S. Barnhart, et al., "Asbestos, asbestosis, and cancer: the Helsinki criteria for diagnosis and attribution," Scandinavian Journal of Work, Environment and Health, vol. 23, no. 4, pp. 311-316, 1997.

[36] American Thoracic Society, "Diagnosis and initial management of nonmalignant diseases related to asbestos," American Journal of Respiratory and Critical Care Medicine, vol. 170, no. 6, pp. 691-715, 2004.

[37] O. Akyol, H. Canatan, H. R. Yilmaz, et al., "PCR/RFLPbased cost-effective identification of SOD2 signal (leader) sequence polymorphism (Ala-9Val) using NgoM IV: a detailed methodological approach," Clinica Chimica Acta, vol. 345, no. 1-2, pp. 151-159, 2004.

[38] V. L. Kinnula, S. Lehtonen, P. Koistinen, et al., "Two functional variants of the superoxide dismutase genes in Finnish families with asthma," Thorax, vol. 59, no. 2, pp. 116-119, 2004.

[39] H. Li, P. W. Kantoff, E. Giovannucci, et al., "Manganese superoxide dismutase polymorphism, prediagnostic antioxidant status, and risk of clinical significant prostate cancer," Cancer Research, vol. 65, no. 6, pp. 2498-2504, 2005.

[40] K. Woodson, J. A. Tangrea, T. A. Lehman, et al., "Manganese superoxide dismutase (MnSOD) polymorphism, $\alpha$ tocopherol supplementation and prostate cancer risk in the alpha-tocopherol, beta-carotene cancer prevention study (Finland)," Cancer Causes and Control, vol. 14, no. 6, pp. 513$518,2003$.

[41] S. Ezzikouri, A. E. El Feydi, A. Chafik, et al., "Genetic polymorphism in the manganese superoxide dismutase gene is associated with an increased risk for hepatocellular carcinoma in HCV-infected Moroccan patients," Mutation Research, vol. 649, no. 1-2, pp. 1-6, 2008.

[42] W. Zhong, L. W. Oberley, T. D. Oberley, T. Yan, F. E. Domann, and D. K. St. Clair, "Inhibition of cell growth and sensitization to oxidative damage by overexpression of manganese superoxide dismutase in rat glioma cells," Cell Growth and Differentiation, vol. 7, no. 9, pp. 1175-1186, 1996.

[43] V. L. Kinnula, U. A. Hodgson, E. K. Lakari, et al., "Extracellular superoxide dismutase has a highly specific localization in idiopathic pulmonary fibrosis/usual interstitial pneumonia," Histopathology, vol. 49, no. 1, pp. 66-74, 2006. 

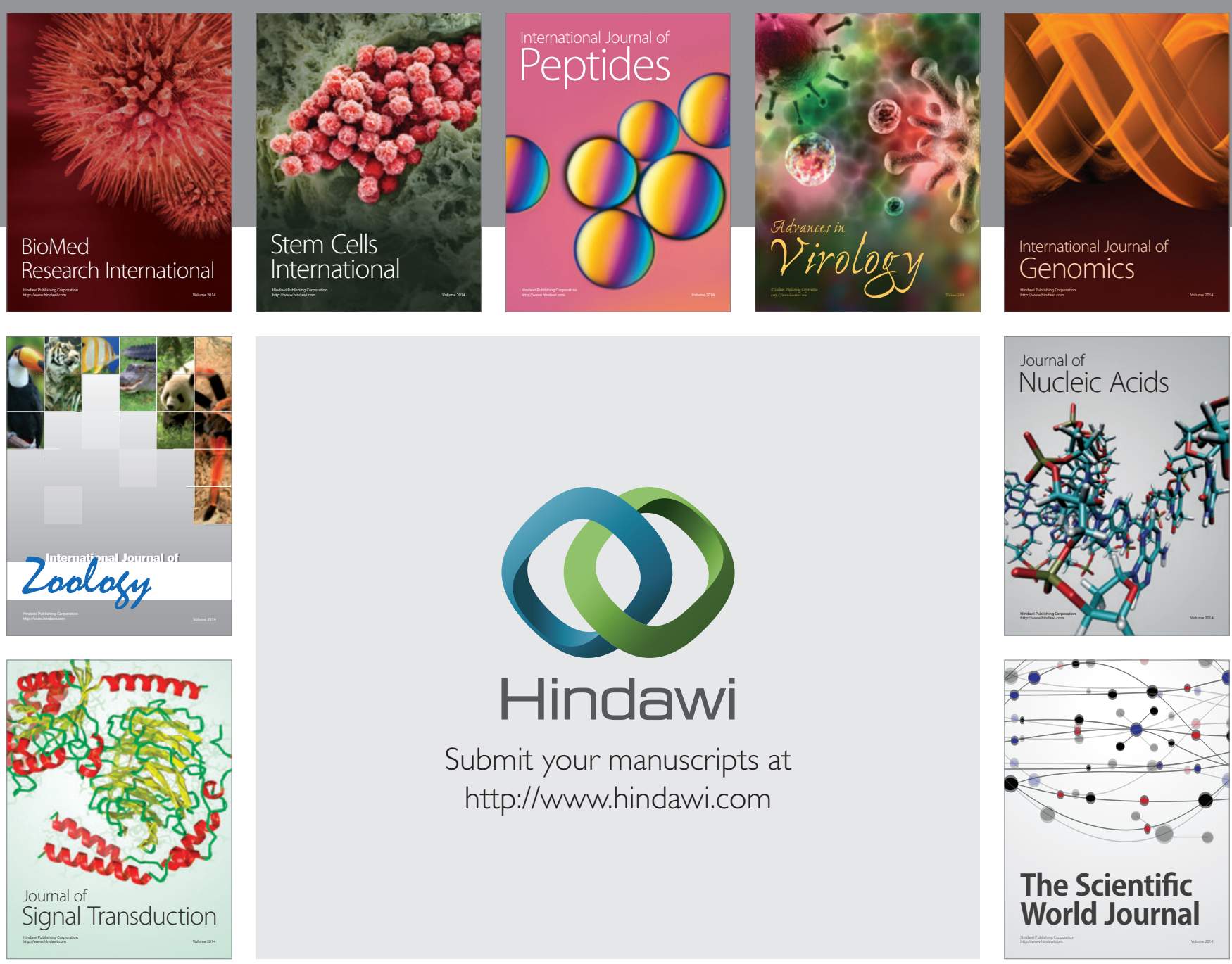

Submit your manuscripts at

http://www.hindawi.com
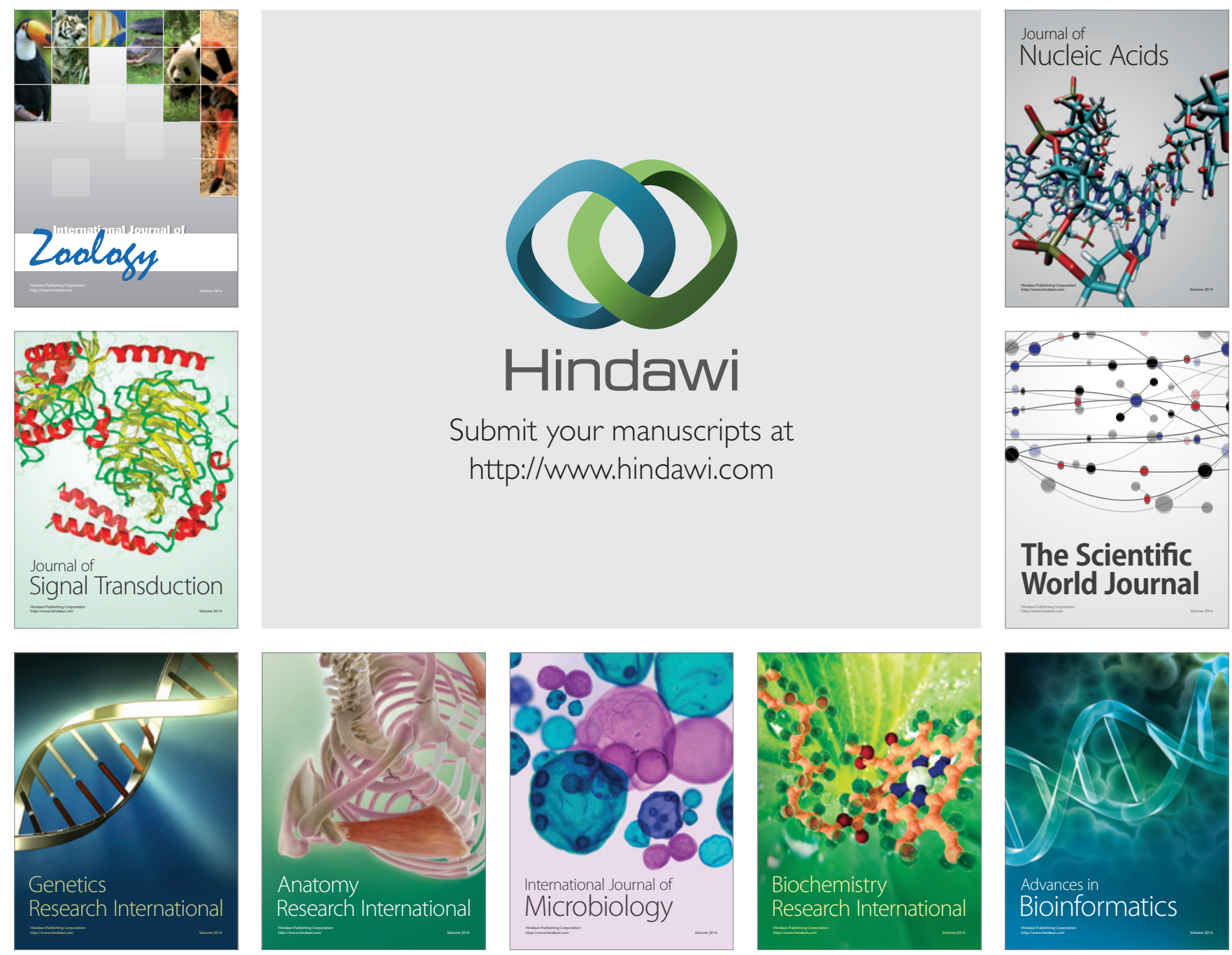

The Scientific World Journal
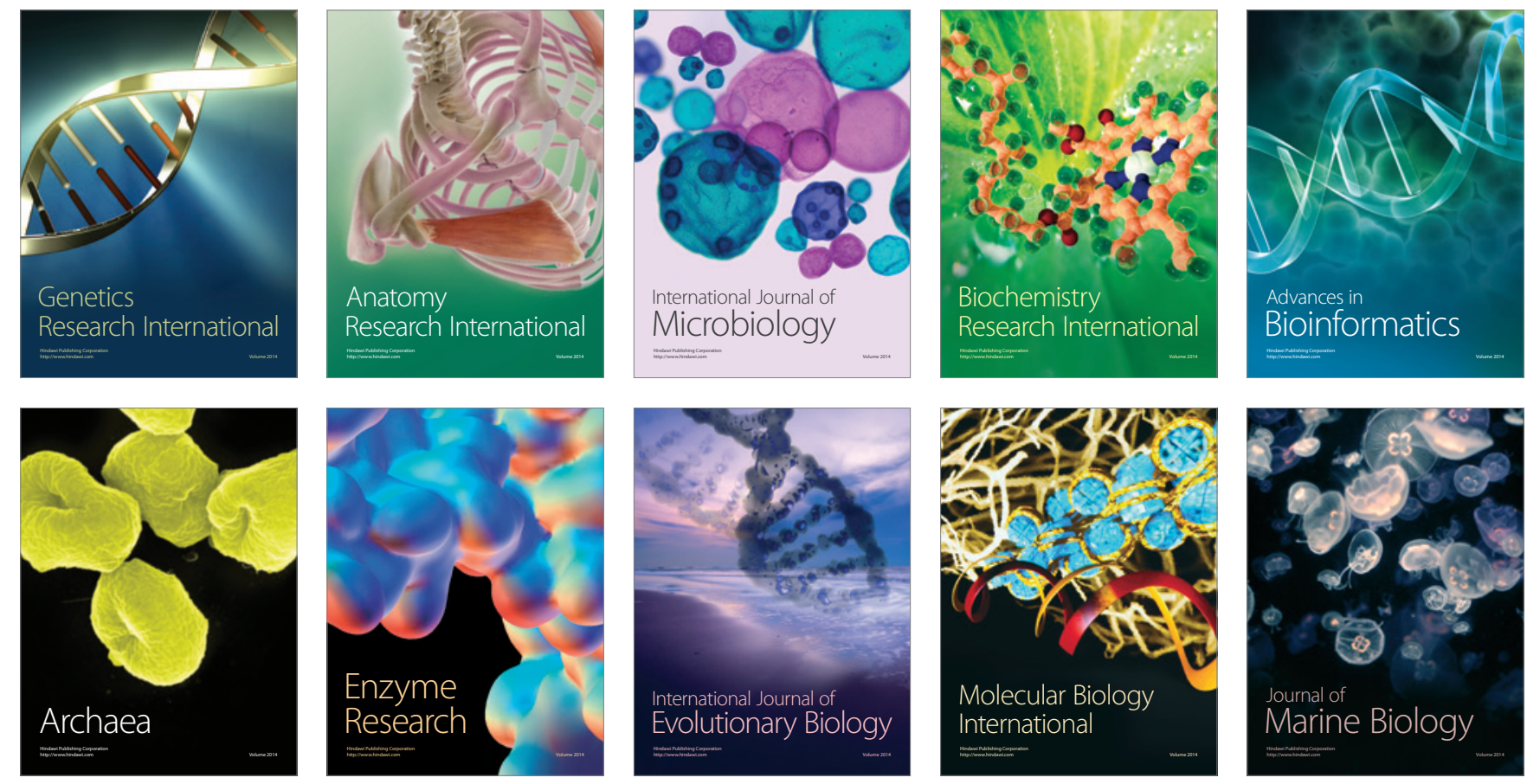